\title{
The Influence of Materials Parameters on Temperature and Stress in Gold Coated Gratings for Pulse Compression
}

\author{
Yi-Han WU ${ }^{1}$, Ke-Peng $W^{1}$, Ping $M A^{2}$, Yun-Ti PU ${ }^{2}$ and Zhi-Lin $X I A^{1, a}$ \\ ${ }^{1}$ State Key Laboratory of Silicate Materials for Architectures, Wuhan University of Technology, \\ Wuhan, Hubei 430070, PR China \\ ${ }^{2}$ Chengdu Fine Optical Engineering Research Center, Chengdu, Sichuan 610041, PR China \\ a Corresponding author 1. E-mail: xiazhilin@whut.edu.cn
}

Keywords: Gold coated gratings; Laser induced damage; Pulse compression gratings.

\begin{abstract}
In this paper, the electromagnetic field, temperature field and stress field distribution of gold coated gratings were calculated to analyze the damage mechanism under ps-pulsed laser irradiation. The finite element method in time domain was used in calculation. The Maxwell's equations were used to calculate the electromagnetic field, the Fourier's law of heat conduction was used to calculate the temperature field and the Hooke's law was used to calculate the stress field. The influences of photoresist parameters on temperature and stress fields in gratings were analyzed. The results reflect that using photoresist which has a high thermal conductivity, low thermal expansion coefficient and low elasticity modulus to fabricate bottom gratings is considered useful for improving damage threshold of gold coated gratings.
\end{abstract}

\section{Introduction}

The chirped pulse amplification (CPA) technology is the main approach to achieve high-intensity short-pulse laser. Diffraction gratings are good candidates for stretching and compressing laser pulses in CPA [1-3]. To achieve high intensity laser pulse, the used pulse compression gratings (PCG) must meet the requirements of broadband high efficiency and high laser damage resistance. The final grating in the pulse compressor needs to endure the irradiation of highest power laser [4, 5]. Metal-coated gratings used for pulse compression were first researched and developed in 1980 [1]. But the rather low damage threshold limits the performances of the CPA laser system [6, 7].

To improve the longevity of a short-pulse laser system, a better understanding of the damage mechanism and constantly improving the laser damage resistance of metal-coated gratings should be given more attention. The usually used electromagnetic field distribution in gratings cannot describe the details of thermal ablation damage in gold coated gratings. To relatively complete describe the damage mechanism of the gold coated gratings, the distributions of temperature field and stress field in gratings caused by laser pulse were calculated in this paper.

\section{Simulations and Discussions}

\section{Simulation Models}

Figure 1(a) shows the used calculation model of gold coated gratings in this paper. A P-polarized laser beam is incident from the left with an incidence angle of $53^{\circ}$, and the vacuum wavelength of it is $800 \mathrm{~nm}$. The laser pulse width is $450 \mathrm{ps}$, and the energy density is $0.67 \mathrm{~J} / \mathrm{cm}^{2}$. Grating material parameters used in calculation are given in Table 1 [8-11]. Considering that the thermal conductivity of a metal will significantly decreased under high temperature, the thermal conductivity of $200 \mathrm{~W} /\left(\mathrm{m}^{*} \mathrm{~K}\right)$ has been taken in calculation. The finite element method in time domain was used in calculation. The Maxwell's equations were used to calculate the electromagnetic field, the Fourier's law of heat conduction was used to calculate the temperature field and the Hooke's law was used to calculate the stress field. 
Table 1 The parameters of materials used in calculation

\begin{tabular}{ccccc}
\hline parameters & air & gold & photoresist & substrate \\
\hline Mass density $-\mathrm{kg} / \mathrm{m}^{3}$ & 1.29 & 19300 & 1180 & 2202 \\
Thermal conductivity $-\mathrm{W} /\left(\mathrm{m}^{*} \mathrm{~K}\right)$ & 0.024 & 200 & 0.18 & 1.38 \\
Heat capacity $-\mathrm{J} /(\mathrm{kg}$ K $)$ & 1011 & 129 & 1470 & 753 \\
Young modulus -GPa & - & 80 & 2.33 & 72 \\
Poisson's ratio & - & 0.42 & 0.34 & 0.17 \\
Coefficient of thermal expansion $-\mathrm{K}^{-1}$ & - & $1.443^{*} 10^{-5}$ & $7.0^{*} 10^{-5}$ & $0.055^{*} 10^{-5}$ \\
Refractivity & 1 & $0.18+5.00 i$ & 1.49 & 1.52 \\
\hline
\end{tabular}

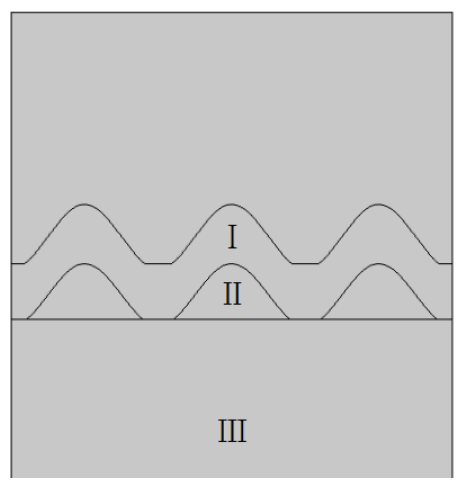

(a)

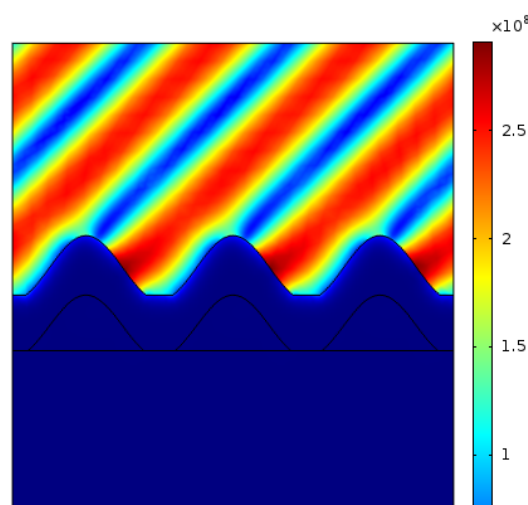

(b)

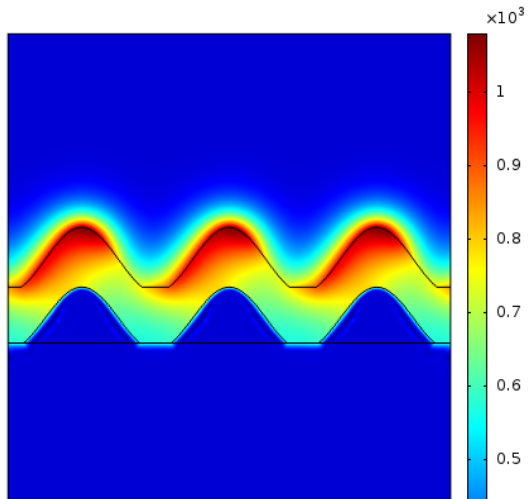

(c)

Figure 1 Schematic diagram, electromagnetic field distribution (unit of $V / m$ ) and temperature field distribution (unit of $K$ ) in gold coated gratings. (a) calculation model of gold coated grating; (b) electromagnetic field distribution; (c) temperature field distribution. The grating structure includes: the metal layer ( I ) with a thickness of $232 \mathrm{~nm}$ and a duty cycle of 0.82 ; the photoresist grating layer ( II ) with a spatial period of $574.7 \mathrm{~nm}$, a duty cycle of 0.79 and a groove depth of $217 \mathrm{~nm}$; and the substrate layer (III) with a thickness of $1200 \mathrm{~nm}$ used in calculation.

In the calculation of the electromagnetic field, laser irradiation is excited on the upper boundary of the calculation domain. The laser irradiation area (with diameter of about 500 micron) contains nearly a thousand grating period. Therefore, the left and right boundaries of the calculation domain were set as periodic boundaries to reduce the computational region, and the same for temperature and stress field calculation.

In the case of nanosecond or sub-nanosecond pulsed irradiation, electrons-lattice heat exchange process can be ignored during the calculation of lattice temperature, and the temperature rise of gold films can be directly calculated according to the absorbed energy [12]. In the calculation of the temperature field, the upper and lower boundaries of the calculation domain are thermal diffusion boundaries. In the calculation of the stress field, the air layer was ignored, the surface of the gold film was set as a free boundary, and the lower boundary of the substrate layer was set as a fixed boundary.

\section{Temperature and Stress Field}

Figure 1(b) shows the electromagnetic field distribution. A standing wave field is formed at the incident side in the air layer. Due to the strong absorption and high reflection characteristics of gold films, the depth that the electromagnetic wave enters into the gold film is extremely limited. Figure 1(c) shows the temperature field distribution. The temperature rise focuses on the surface (the surface refers to the gold film surface). As for the layers of the photoresist and the substrate, due to the short thermal diffusion time and their negligible absorption coefficient, the temperature rises of them are very small.

The calculating stress and strain fields are shown in Fig. 2. The stress (Von Mises equivalent stress) in gold films mainly focuses on the groove surface. The surface peak stress locates in the junction region of grating ridges and grooves. The interface (the interface refers to the interface between gold films and photoresist ratings) stress mainly focuses around the photoresist grating top. 
Moreover, the stress in the photoresist layer and the stress at the photoresist-substrate interface are relatively small, so damages rarely originate from these locations if thermal stress dominates the damage process.

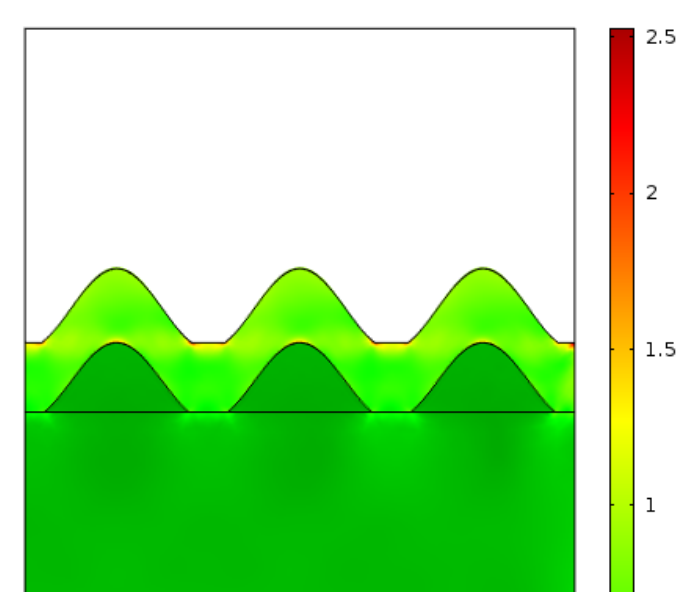

(a)

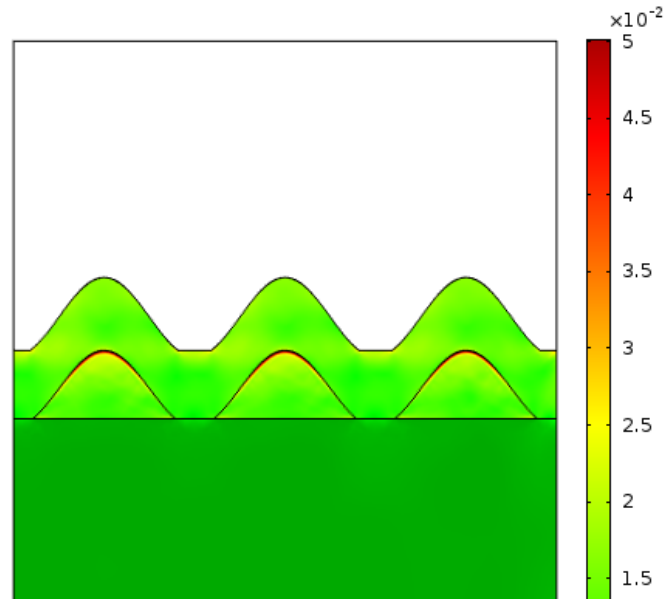

(b)

Figure 2 The stress field and strain field distribution in gold coated gratings. (a) stress field distribution (unit of $G P a$ ); (b) strain field distribution

\section{The Influence of Materials Parameters}

The picoseconds pulsed laser induced damage is mainly dominated by the thermal and thermal stress processes. Thus, the variation of material thermodynamic parameters, especially the photoresist thermodynamic parameters, will change the damage threshold, damage morphology and damage process. First of all, thermal stress distributions have been calculated by using the laser energy density of $0.85 \mathrm{~J} / \mathrm{cm}^{2}$, changing the thermal expansion coefficient of photoresist from 0.5 to $40.5 \times 10^{-6} / K$, adopting the step of $1.0 \times 10^{-5} / K$, and keeping the other parameters unchanged. The calculation results are shown in Fig.3. It can be seen that the thermal expansion coefficient of photoresist has a little influence on the surface stress and strain as well as the interface stress. However, it has a significant influence on the interface strain, and the interface peak strain increases significantly with the increasing of the thermal expansion coefficient of photoresist.

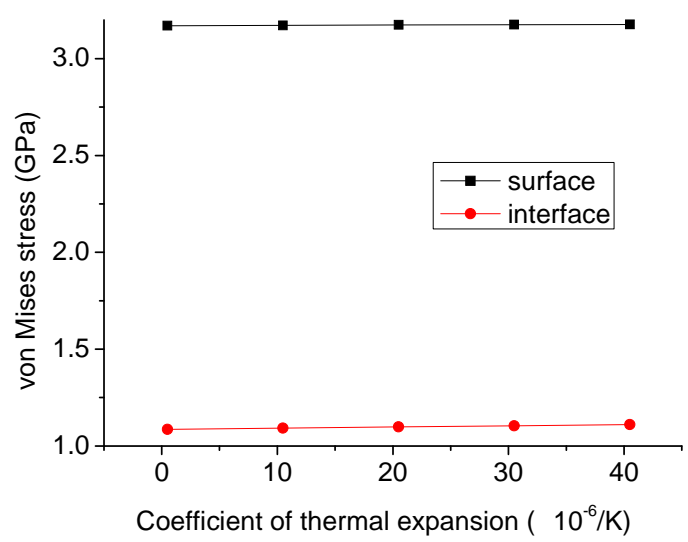

(a)

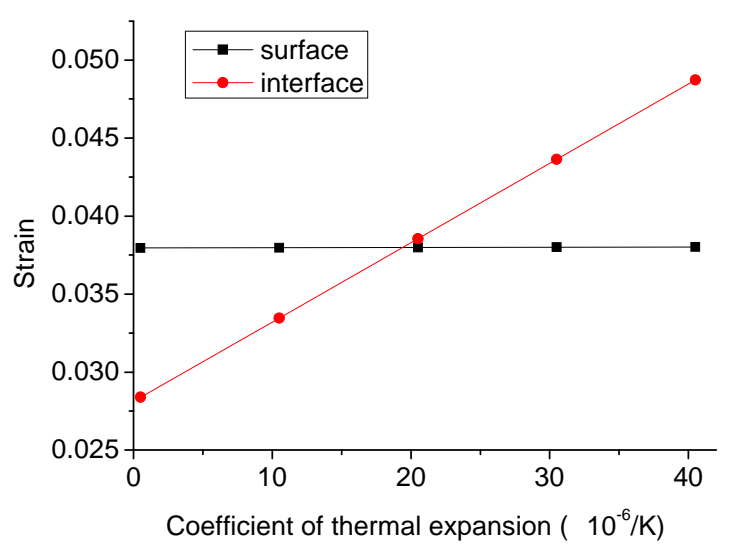

(b)

Figure 3 The peak stress and strain vary with the thermal expansion coefficient of photoresist. (a) peak stress; (b) peak strain 


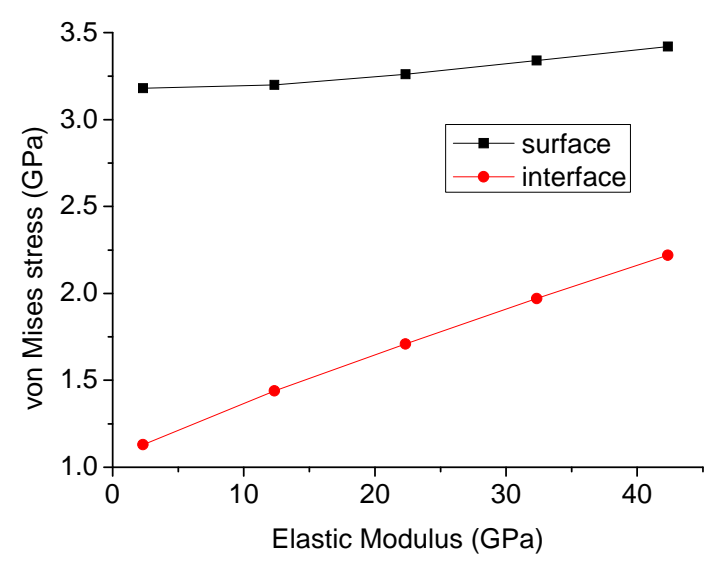

(a)

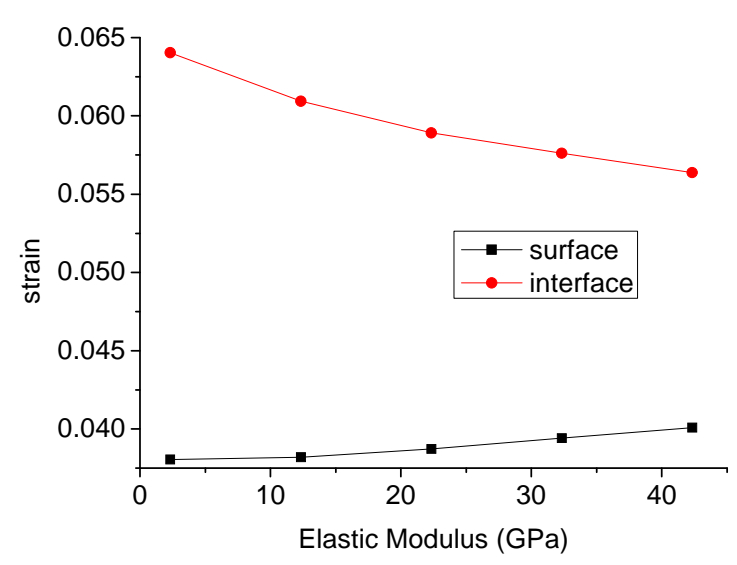

(b)

Figure 4 The peak stress and strain vary with the elasticity modulus of photoresist. (a) peak stress;

(b) peak strain

Then, thermal stress distributions have been calculated by using laser energy density of $0.85 \mathrm{~J} / \mathrm{cm}^{2}$, changing the elasticity modulus of photoresist from 2.33 to $42.33 \mathrm{GPa}$, adopting the step of 10.0GPa, and the calculation results are shown in Fig.4. It can be seen that the elasticity modulus of photoresist has a little influence on the surface stress and strain. The surface peak stress and strain slightly increase with the increasing of the elasticity modulus of photoresist. However, the elasticity modulus of photoresist has a great influence on the interface stress and strain. The interface peak stress increases significantly and the interface peak strain decreases significantly with the increasing of the elasticity modulus of photoresist.

In addition, temperature and thermal stress distributions have been calculated by using laser energy density of $0.85 \mathrm{~J} / \mathrm{cm}^{2}$, changing the thermal conductivity of photoresist from 0.18 to 8.18 $W /\left(m^{*} K\right)$, adopting the step of $9.0 \mathrm{~W} /\left(m^{*} K\right)$, and the calculation results are shown in Fig.5. It can be seen that the surface and interface temperatures have different degrees of decline, and the interface temperature decreases more obviously. In the meantime, the variations of the surface stress and strain as well as the variation of the interface stress are relatively small with the increasing of the thermal conductivity of photoresist. However, the interface peak strain decreases significantly. 


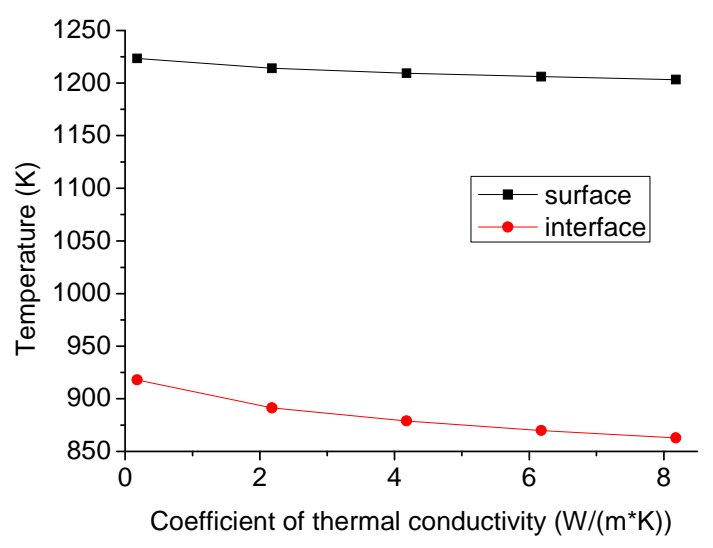

(a)

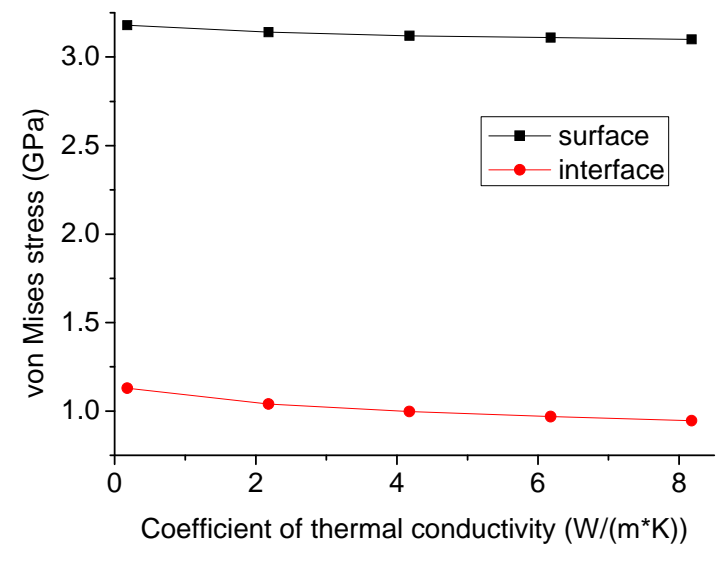

(b)

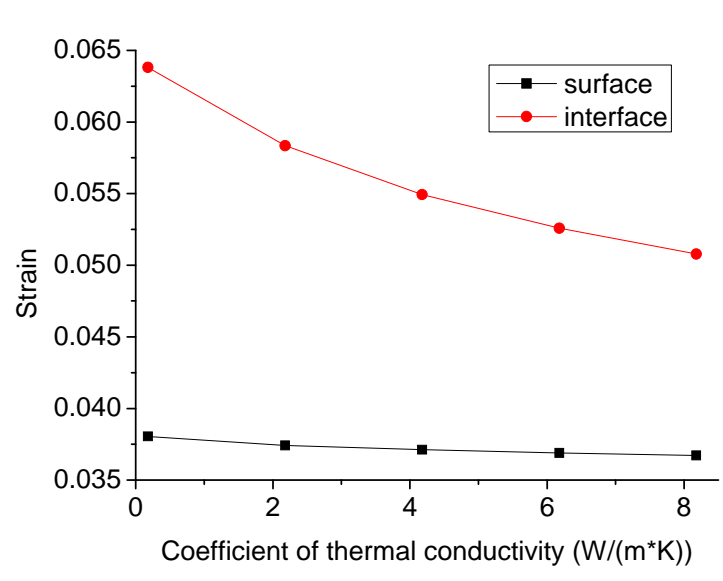

(c)

Figure 5 The peak temperature, stress and strain vary with the thermal conductivity of photoresist. (a) peak temperature; (b) peak stress; (c) peak strain

The thermal expansion coefficient, elasticity modulus and thermal conductivity of photoresist have different degrees of influence on the temperature, stress and strain peaks. Using different photoresist can change the gratings damage threshold and the damage characteristic when design the gold coated gratings structure. Thus, the gratings laser damage threshold can be improved by decreasing the temperature, stress and strain values inside the gratings when the damage process is dominated by thermal and thermodynamic actions, such as increasing the thermal conductivity and decreasing the elasticity modulus or the thermal expansion coefficient of the under layer gratings (usually is photoresist). The next step of our work will focus on choosing photoresist from these aspects for improving gratings damage threshold.

\section{Conclusions}

The electromagnetic field, temperature field, stress and strain field of gold coated gratings under the picoseconds pulsed laser irradiation were calculated, and the influences of photoresist parameters on temperature and stress fields in gratings were analyzed. The thermal expansion coefficient, elasticity modulus and thermal conductivity of photoresist have different degrees of influence on the temperature, stress and strain field. The photoresist which has a high thermal conductivity, low thermal expansion coefficient and low elasticity modulus can be chose to fabricate bottom gratings for improving the laser damage resistance. 


\section{Acknowledgments}

The work was supported by NSAF (No. U1430121). The authors also gratefully acknowledge the financial support of the Open Research Fund of Key Laboratory of Material for High Power Lasers - Chinese Academy of Sciences.

\section{References}

[1] D. Strickland and G. Mourou, Compression of amplified chirped optical pulses, Opt. Comm. 56 (1985) 219-221.

[2] M. Rumpel, M. Moeller, C. Moormann, T. Graf, A. M. Abdou, Broadband pulse compression gratings with measured 99.7\% diffraction efficiency. Opt. Lett. 39 (2014) 323-326.

[3] P. Poole, S. Trendafilov, G. Shvets, D. Smith, E. Chowdhury, Femtosecond laser damage threshold of pulse compression gratings for petawatt scale laser systems, Opt. Express 21 (2013) 26341-26351.

[4] J. Neauport, N. Bonod, S. Hocquet, S. Palmier, and G. Dupuy, Mixed metal dielectric gratings for pulse compression, Opt. Express 18 (2010) 23776-23783.

[5] Y. Du, X. Wu, M. Zhu, Z. Le, Theoretical and experimental research on laser-induced damage of cylindrical subwavelength grating. Opt. Express 23 (2015) 24296-24307.

[6] H. Guan, Y. Jin, S. Liu, F. Kong, Y. Du, Near-field optical properties of wide bandwidth metal multi-layer dielectric gratings for pulse compressor, Appl. Phys. B 114 (2014) 557-565.

[7] S. Liu, J. Ma, Z. Shen, Y. Jin, J. Shao, and Z. Fan, Optimization of thin-film design for multi-layer dielectric gratings, Appl. Surf. Sci. 253 (2007) 3642-3648.

[8] P. B. Johnson, R. W. Christy, Optical Constants of the Noble Metals, Phys. Rev. B 6 (1972) 4370 .

[9] http://www.crystran.co.uk/optical-materials/silica-glass-sio2.

[10] http://www.newworldencyclopedia.org/entry/Gold.

[11] J. K., The thermal conductivity of gold, Gold Bull. 11 (1978) 73-73.

[12] E. G. Gamaly, The physics of ultra-short laser interaction with solids at non-relativistic intensities, Phys. Reports 508 (2011) 91-243. 